\title{
FIFO scheduling of divisible loads with return messages under the one-port model
}

\author{
Olivier Beaumont ${ }^{1}$, Loris Marchal ${ }^{2}$, Veronika Rehn ${ }^{2}$ and Yves Robert ${ }^{2}$ \\ 1: LaBRI, UMR CNRS 5800 \\ Bordeaux, France \\ Olivier.Beaumont@labri.fr \\ 2: LIP, UMR CNRS-INRIA-UCBL 5668 \\ ENS Lyon, France \\ \{Loris. Marchal, Veronika. Rehn, \\ Yves.Robert\}@ens-lyon.fr
}

\begin{abstract}
This paper deals with scheduling divisible load applications on star networks, in presence of return messages. This work is a follow-on of [6, 7], where the same problem was considered under the two-port model, where a given processor can simultaneously send and receive a message. Here, we concentrate on the one-port model, where a processor can either send or receive a message at a given time step. The problem of scheduling divisible load on star platforms turns out to be very difficult as soon as return messages are involved. Unfortunately, we have not been able to assess its complexity, but we provide an optimal solution in the special (but important) case of FIFO communication schemes. We also provide an explicit formula for the optimal number of load units that can be processed by a FIFO ordering on a bus network. Finally, we provide a set of MPI experiments to assess the accuracy and usefulness of our results in a real framework.
\end{abstract}

\section{Introduction}

This paper deals with scheduling divisible load applications [9] on heterogeneous platforms. We target a master-worker implementation where the master initially holds (or generates data for) a large amount of work that will be executed by the workers. In the end, output results will be returned by the workers to the master. Each worker has a different computational speed, and each master-worker link has a different bandwidth, thereby making the (single-level tree) platform fully heterogeneous.

The divisible load scheduling problem (DLS) here is first to decide how many load units the master sends to each worker, and in which order. After receiving its share of the data, each worker executes the corresponding work and returns the results to the master. Again, the ordering of the return messages must be decided by the scheduler. From a theoretical standpoint, the success of the DLS model is mostly due to its analytical tractability. Optimal algorithms and closed-form formulae exist for important instances of the divisible load problem. However, adding return messages dramatically complicates the picture, while it is a very natural extension in practice. Indeed, without return messages, it is implicitly assumed that the size of the results to be transmitted to the master after the computation is negligible, and hence has no (or very little) impact on the whole DLS problem. This may be realistic for some particular DLS applications, but not for all of them. For example, suppose that the master is distributing files to the workers. After processing a file, the worker will typically return results in the form of another file, possibly of shorter size, but still non-negligible. In some situations, the size of the return message may even be larger than the size of the original message: for instance the master initially scatters instructions on some large computations to be performed by each worker, such as the generation of several cryptographic keys; in this case each worker would receive a few bytes of control instructions and would return longer files containing the keys.

Because it is very natural and important in practice, several authors have investigated the problem with return messages: see the papers $[4,15,24,3,1]$. However, all the results obtained so far are very partial. Intuitively, there are hints that suggest that the problem with return messages is much more complicated. The first hint lies in the combinatorial space that is open for searching the best solution. There is no reason for the ordering of the initial messages sent by the 
master to be the same as the ordering for the messages returned to the master by the workers after the execution. In some situations a FIFO strategy (the worker first served by the master is the first to return results, and so on) may be preferred, because it provides a smooth and well-structured pipelining scheme. In [1], Adler, Gong and Rosenberg show that all FIFO strategies are equally performing on a bus network, but even the analysis of FIFO strategies is a difficult open problem on a star network.

This work is a follow-on of $[6,7]$ where we have studied FIFO strategies under the two-port model, where the master can simultaneously send data to one worker and receive from another. In this paper, we study FIFO strategies under the one-port model, where the master can only be enrolled in a single communication at any time-step. The one-port model turns to be be more complicated to analyze, because of the additional constraint imposed on the communication medium. However, it is also more realistic in practice, and all the MPI experiments reported in Section 3 obey this model.

As pointed out, adding return messages dramatically complicates the search for an optimal solution, despite the simplicity of the linear cost model. In fact, we show that the best FIFO schedule may well not involve all processors, which is in sharp contrast with previous results from the literature. The main contributions of this paper are the characterization of the best FIFO strategy on a star network, together with an experimental comparison of several FIFO orderings. Rather than simulations $[6,7]$ we perform extensive MPI experiments on heterogeneous platforms.

The rest of the paper is organized as follows: we develop our theoretical framework and results in Section 2 and we present our MPI experiments in Section 3, then we briefly survey related work in 4 and we state some concluding remarks in Section 5.

\section{Theoretical results}

\subsection{Target platform and model}

As illustrated in Figure 1, we target a star network $\mathcal{S}=\left\{P_{0}, P_{1}, P_{2}, \ldots, P_{p}\right\}$, composed of a master $P_{0}$ and of $p$ workers $P_{i}, 1 \leq i \leq p$. In the linear cost model, each worker $P_{i}$ has a (relative) computing power $w_{i}$ : it takes $X . w_{i}$ time units to execute $X$ units of load on worker $P_{i}$. Similarly, it takes $X . c_{i}$ time units to send the initial data needed for computing $X$ units of load from $P_{0}$ to $P_{i}$, and $X . d_{i}$ time units to return the corresponding results from $P_{i}$ to $P_{0}$. Note that a bus network is a star network such that all communication links have the same characteristics: $c_{i}=c$ and $d_{i}=d$ for each worker $P_{i}, 1 \leq i \leq p$. It is natural to assume that the quantity $\frac{d_{i}}{c_{i}}$ is a constant $z$ that depends on the application but not on the selected worker. In other words, workers who communicate faster with the master for the initial message will also communicate faster for the return message.

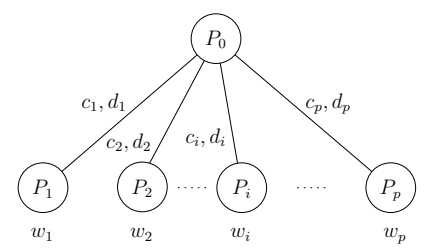

Figure 1. Targeted platform

The standard one-port model in DLS problems for communications is defined as follows: (i) the master can only send data to (and receive data from) a single worker at a given time-step; (ii) a given worker cannot start its execution before it has terminated the reception of the message from the master; similarly, it cannot start sending the results back to the master before finishing the computation. In fact, this one-port model naturally comes in two flavors with return messages, depending upon whether we allow the master to simultaneously send and receive messages or not. If we do allow for simultaneous sends and receives, we have the two-port model we have studied in the companion paper [7]. Here we concentrate on the fully one-port model, where the master cannot be enrolled in more than one communication at any time-step.

\subsection{Scheduling problem}

We focus on the following problem: given a time bound $T$, what is the maximum number of load units that can be processed within time $T$ ? As we adopt the classical linear cost model (communication and computation costs are proportional to the number of load units), we can consider that $T=1$ without loss of generality. Also, owing to the linearity of the model, this problem is equivalent to the problem of minimizing the execution time for a given amount of load to be processed. A solution to the scheduling problem is characterized by the subset of enrolled processors, and their respective loads (we let $\alpha_{i}$ denote the amount of load assigned to, and processed by, each participating worker $P_{i}$ ), together with the dates at which each event (incoming communication, computation, return message) starts on each processor.

We can make a few useful simplifications. First, we can assume that each worker starts computing right 
upon completion of the initial communication from the master. Also, we can assume that the master sends initial messages as soon as possible, i.e. without any delay between two consecutive messages. Symmetrically, we can assume that return messages are sent consecutively, and as late as possible, to the master. However, some idle time may well occur between the time at which a worker $P_{i}$ has completed its work and the time at which it starts returning the message, just because the master may be busy communicating with another worker. This idle time for $P_{i}$ will be denoted as $x_{i}$. We can now sum up the description of a schedule by a first permutation $\sigma_{1}$ representing the ordering of sending operations, from the master to each worker, a second permutation $\sigma_{2}$ representing the ordering of receiving operations, from each worker to the master, the quantity $\alpha_{i}$ of load units sent to each worker $P_{i}$, and the idle time $x_{i}$ of each worker $P_{i}$.

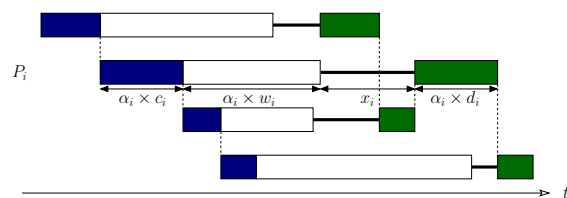

The permutation for input messages is $\sigma_{1}=$ $(1,2,3,4)$, while the permutation for output messages is $\sigma_{2}=(1,3,2,4)$.

Figure 2. Example of a general schedule.

This knowledge allows us to completely describe a schedule, as represented in Figure 2. We have not been able to assess a general complexity result, but we have explored special instances of the scheduling problem, namely FIFO schedules for which the order for result messages is the same as the order for input data messages $\left(\sigma_{2}=\sigma_{1}\right)$ : the first worker receiving its data is also the first to send back its results. Figure 3(a) gives an example of such a FIFO schedule.

Note that when the ordering for results messages is the reverse of the order for input data messages $\left(\sigma_{2}=\sigma_{1}^{R}\right)$, we have a LIFO schedule: the first worker receiving its data is the last to send back its results. Figure $3(\mathrm{~b})$ presents an example of such a LIFO schedule. All LIFO schedules naturally obey the one-port model, and we refer to $[6,7]$ for a characterization of the optimal LIFO schedule.

\subsection{Linear Program for a given scenario}

In this section, we show that a linear programming approach can be used to compute the throughput, once the set of participating processors and the ordering of

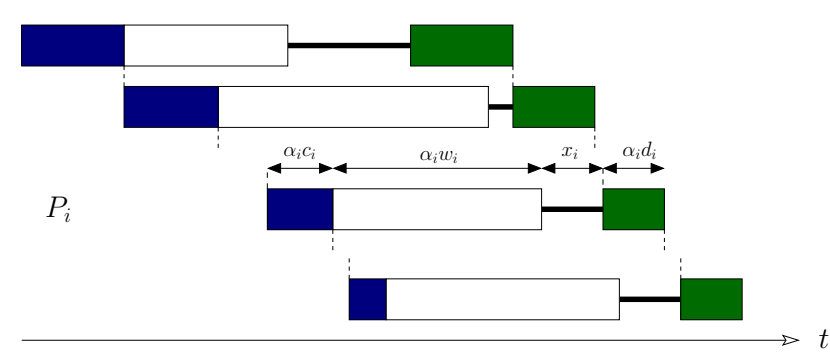

(a) FIFO schedule

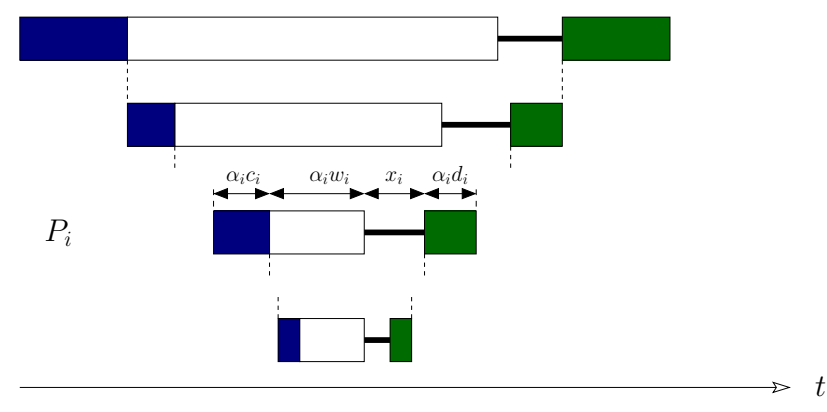

(b) LIFO schedule

Figure 3. Examples of special scenarios.

the messages (permutations $\sigma_{1}$ and $\sigma_{2}$ ) have been determined. We illustrate this approach for a FIFO solution, because we mainly concentrate on FIFO schedules in this paper. However, the method can easily be extended to any permutation pair.

Consider the FIFO schedule represented on Figure 3(a), where $q$ processors numbered $P_{1}$ to $P_{q}$ are enrolled in the computation. Consider processor $P_{i}$. Before receiving its initial data, $P_{i}$ has to wait for all previous data transfers, i.e. the time needed for the master to send $\alpha_{j}$ load units to each processor $P_{j}$, for $j<i$; this takes $\sum_{j=1}^{i-1} \alpha_{j} \times c_{j}$ time-steps. Then processor $P_{i}$ receives its data in $\alpha_{i} \times c_{i}$ time-steps, and processes it in $\alpha_{i} \times w_{i}$ time-steps. Next, $P_{i}$ possibly waits for the communication medium to be free, during $x_{i}$ time-steps. Finally, processor $P_{i}$ sends back its results to the master, in $\alpha_{i} \times d_{i}$ time-steps. There remains to wait for processors $P_{i+1}$ to $P_{q}$ to send their results to the master, which requires $\sum_{j=i+1}^{q} \alpha_{j} \times d_{j}$ time-steps. Altogether, all this has to be done within a time less than the total execution time $T=1$, hence we derive the constraint:

$$
\sum_{j=1}^{i} \alpha_{j} \times c_{j}+\alpha_{i} \times w_{i}+\sum_{j=i}^{q} \alpha_{j} \times d_{j}+x_{i} \leq 1
$$


To enforce one-port constraints, we have to ensure that communications do not overlap, which translates into:

$$
\sum_{i=1}^{q} \alpha_{i} \times c_{i}+\sum_{i=1}^{q} \alpha_{i} \times d_{i} \leq 1
$$

The throughput $\rho$ of the schedule the total number of tasks processed in time $T=1: \rho=\sum_{i=1}^{q} \alpha_{i}$.

To sum it up, given a scenario consisting of a set of $q$ participating processors and two permutations for initial and back communications, we can compute the optimal throughput and derive a schedule achieving such a throughput by solving the following linear program:

$$
\begin{aligned}
& \text { MAXimize } \rho=\sum_{i=1}^{q} \alpha_{i}, \\
& \text { Under THE CONSTRAINTS } \\
& \qquad \begin{array}{c}
\forall i=1, \ldots q, \\
(2 \mathrm{a}) \quad \sum_{j=1}^{i} \alpha_{j} c_{j}+\alpha_{j} w_{j}+\sum_{j=i}^{q} \alpha_{j} d_{j}+x_{i} \leq 1 \\
(2 \mathrm{~b}) \quad \sum_{i=1}^{q} \alpha_{i} \times c_{i}+\sum_{i=1}^{q} \alpha_{i} \times d_{i} \leq 1 \\
(2 \mathrm{c}) \quad \forall i=1, \ldots q, \quad \alpha_{i} \geq 0 \\
(2 \mathrm{~d}) \quad \forall i=1, \ldots q, \quad x_{i} \geq 0
\end{array}
\end{aligned}
$$

For a given scenario, the cost of this linear programming approach may be acceptable. However, as already pointed out, there is an exponential number of scenarios. Worse, there is an exponential number of FIFO scenarios, even though there is a single permutation to try in this case.

\subsection{Optimal FIFO schedule on a star plat- form}

In this section, we analyze FIFO schedules. We assume that $d_{i}=z c_{i}$ for $1 \leq i \leq p$, with $0<z<1$. The case $z>1$ is symmetrical (see [5]). The following theorem summarizes the main result:

Theorem 1. There exists an optimal one-port FIFO schedule where:

- processors are ordered in non-decreasing value of $c_{i}$; - all processors taking part in the computation have no idle time, except possibly the last one.

Therefore, an optimal FIFO schedule (with resource selection) can be determined in polynomial time.

We start the proof with some preliminary lemmas. For a while, we assume that the set of participating processors has been determined, and we come back to this resource selection problem later. The following lemmas provide useful characterizations of an optimal solution:
Lemma 1. There exists an optimal FIFO one-port schedule where at most one participating processor has some idle time.

Proof. Let us assume that there are $q$ processors participating in the solution. Any optimal FIFO oneport schedule is an optimal solution to the linear program (2). In this linear program, there are $2 q$ unknowns (the $\alpha_{i}$ 's and the $x_{i}$ 's) and $3 q+1$ constraints. Using linear programming theory [25], we know that there is a vertex of the polyhedron defined by the constraints which is optimal. At this optimum, there are $2 q$ out of the $3 q+1$ constraints which are tight, i.e. which are equalities. Since we are considering $q$ workers participating to the processing, none of the $\alpha_{i} \geq 0$ constraints is an equality. So, at this optimal vertex, there are $2 q$ equalities among the remaining $2 q+1$ constraints: in other words, at most one constraint is not tight. In particular, there exists at most one processor $P_{i}$ with $x_{i}>0$, which means that there is at most one processor with idle time, what achieves the proof of Lemma 1.

Lemma 2. There exists an optimal FIFO one-port schedule where only the last participating processor may have some idle time.

Proof. Let $\mathcal{S}$ be an optimal FIFO one-port schedule, and assume that it involves processors $P_{1}$ to $P_{q}$ in this order. According to Lemma 1, there is at most one processor that may have some idle time between the end of its processing and the start of its backward communication. By contradiction, suppose that this processor is $P_{i}$, where $i<q$ and $x_{i}>0$. We focus on processors $P_{i}$ and $P_{j}=P_{i+1}$. Their activity in $\mathcal{S}$ is represented on Figure 4. We aim at building a schedule $\mathcal{S}^{\prime}$,

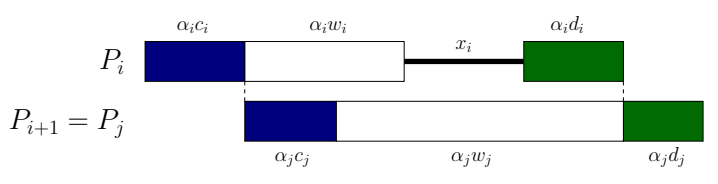

Figure 4. Activity of $P_{i}$ and $P_{i+1}$ in the initial schedule $\mathcal{S}$

where the $i$-th participating processor has no idle time, but the $i+1$-th participating processor may have idle time, and the number of tasks processed in $\mathcal{S}^{\prime}$ is not smaller than the number of tasks processed in $\mathcal{S}$.

We consider two different cases, according to the communication speeds of $P_{i}$ and $P_{j}$ :

- Case $1, c_{i} \leq c_{j}$.

The transformation used to analyze this case is 


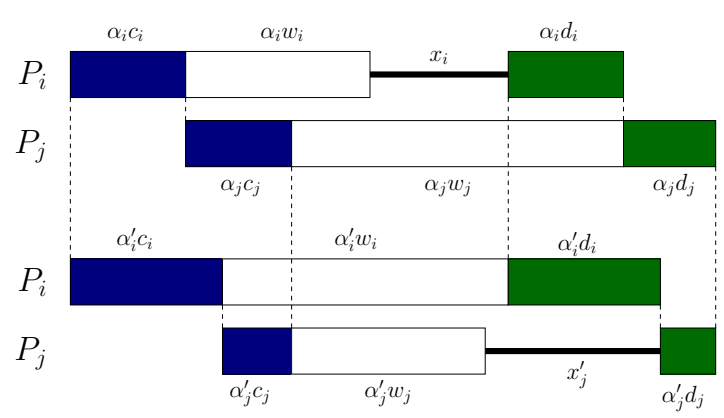

Figure 5. From $\mathcal{S}$ to $\mathcal{S}^{\prime}$, in the case $c_{i} \leq c_{j}$

depicted in Figure 5. Roughly, we keep the overall communication time and the time intervals used for forward and backward communications unchanged, so that other communications (with $\left.P_{k} \neq P_{i}, P_{j}\right)$ are not affected by the transformation. We increase the amount of tasks processed by $P_{i}$, and decrease the amount of tasks processed by $P_{j}$, so that the overall number of tasks processed by $P_{i}$ and $P_{j}$ increases.

More precisely, let us set

$$
\begin{aligned}
\alpha_{i}^{\prime}=\alpha_{i}+\frac{x_{i}}{c_{i}+w_{i}}, & \alpha_{j}^{\prime}=\alpha_{j}-\frac{c_{i}}{c_{j}} \frac{x_{i}}{c_{i}+w_{i}}, \\
\alpha_{k}^{\prime}=\alpha_{k} & \text { for all } k \neq i, j
\end{aligned}
$$

We set $x_{i}^{\prime}=0$, and we will formulate the $x_{j}$ value later. The total time needed for data transfers in the new schedule $\mathcal{S}^{\prime}$ is given by

$$
\begin{aligned}
c_{i} \alpha_{i}^{\prime}+c_{j} \alpha_{j}^{\prime} & =c_{i} \alpha_{i}+c_{j} \alpha_{j}+c_{i} \frac{x_{i}}{c_{i}+w_{i}}-c_{j} \frac{c_{i}}{c_{j}} \frac{x_{i}}{c_{i}+w_{i}} \\
& =c_{i} \alpha_{i}+c_{j} \alpha_{j}
\end{aligned}
$$

Therefore, the overall communication time for sending data messages to $\left(P_{i}, P_{j}\right)$ is the same for $\mathcal{S}$ and $\mathcal{S}^{\prime}$. In other words, $P_{j}$ starts computing at the same date in $\mathcal{S}$ and in $\mathcal{S}^{\prime}$. Since $d_{i}=z c_{i}$, we also have

$$
\begin{aligned}
d_{i} \alpha_{i}^{\prime}+d_{j} \alpha_{j}^{\prime} & =z\left(c_{i} \alpha_{i}^{\prime}+c_{j} \alpha_{j}^{\prime}\right)=z\left(c_{i} \alpha_{i}+c_{j} \alpha_{j}\right) \\
& =d_{i} \alpha_{i}+d_{j} \alpha_{j},
\end{aligned}
$$

so that the overall time for backward communications is also the same.

The time between the start of the data transfer for $P_{i}$ and the start of its return transfer is given by

$$
\begin{aligned}
& \text { in } \mathcal{S}:\left(c_{i}+w_{i}\right) \alpha_{i}+x_{i} \\
& \text { in } \begin{aligned}
\mathcal{S}^{\prime}:\left(c_{i}+w_{i}\right) \alpha_{i}^{\prime} & =\left(c_{i}+w_{i}\right)\left(\alpha_{i}+\frac{x_{i}}{c_{i}+w_{i}}\right) \\
& =\left(c_{i}+w_{i}\right) \alpha_{i}+x_{i}
\end{aligned}
\end{aligned}
$$

So the transfer of the return message for $P_{i}$ starts at the same date in $\mathcal{S}$ and $\mathcal{S}^{\prime}$. Together with the fact that communication times are identical in $\mathcal{S}$ and $\mathcal{S}^{\prime}$ for the group $\left(P_{i}, P_{j}\right)$, this ensures that we do not perturb the rest of the execution: nothing changes in the data transfers before the date when $P_{i}$ starts receiving, and after $P_{j}$ stops receiving. The same holds true for transferring results back to the master.

In $\mathcal{S}^{\prime}, P_{j}$ has fewer tasks to process than in $\mathcal{S}$, but it can start computing at the same date (since the total data transfer time is the same), and it can stop working later, as its return transfer is shorter. Therefore, we need to introduce some idle time $x_{j}^{\prime}$ on $P_{j}$. Since the time between the start of its computation and the end of its output transfer is the same for $\mathcal{S}$ and $\mathcal{S}^{\prime}$, we can write:

$$
\begin{aligned}
\left(w_{j}+d_{j}\right) \alpha_{j}^{\prime}+x_{j}^{\prime} & =\left(w_{j}+d_{j}\right) \alpha_{j} \\
\left(w_{j}+d_{j}\right) \times \frac{-c_{i}}{c_{j}} \frac{x_{i}}{c_{i}+w_{i}}+x_{j}^{\prime} & =0 \\
x_{j}^{\prime} & =x_{i}\left(\frac{c_{i}}{c_{j}} \frac{w_{j}+d_{j}}{c_{i}+w_{i}}\right)
\end{aligned}
$$

Thus, $x_{j}^{\prime}>0$ as soon as $c_{i}>c_{j}$, and $x_{j}^{\prime}=0$ otherwise $\left(c_{i}=c_{j}\right)$. The new schedule $\mathcal{S}^{\prime}$ is represented in Figure 5, where it is compared to $\mathcal{S}$. The amount of tasks processed by both $P_{i}$ and $P_{j}$ in $\mathcal{S}^{\prime}$ is given by

$\sum_{i} \alpha_{i}^{\prime}=\sum_{i} \alpha_{i}+\epsilon_{i}-\epsilon_{j}=\sum_{i} \alpha_{i}+\frac{c_{j}-c_{i}}{c_{j}} \frac{x_{i}}{c_{i}+w_{i}}$.

Since $c_{j} \geq c_{i}, \mathcal{S}^{\prime}$ processes at least as many tasks as $\mathcal{S}$ and we have moved the gap one step further from $P_{i}$ to $P_{j}$.

- case $2, c_{i}>c_{j}$.

In this case, it is not worth moving the gap from $P_{i}$ to $P_{j}$. Therefore, the transformation (see Figure 6) consists in keeping the gap at $P_{i}$, while changing the communication ordering of the FIFO schedule, by switching $P_{i}$ and $P_{j}$. The sketch of the proof is essentially the same as in the first case. We choose the transformation so that the overall communication time for both forward and backward communications to $P_{i}$ and $P_{j}$ is the same, thus letting other communications unchanged. Then, we prove that such a transformation increases the overall number of tasks processed by $P_{i}$ and $P_{j}$. More precisely, after the exchange, the number of tasks processed by each processor is the following:

$$
\begin{aligned}
\alpha_{j}^{\prime} & =\alpha_{j}+\frac{\alpha_{i} c_{i}(1-z)}{c_{j}+w_{j}} \\
\alpha_{i}^{\prime} & =\alpha_{i}-\frac{\alpha_{i} c_{j}(1-z)}{c_{j}+w_{j}} \\
\alpha_{k}^{\prime} & =\alpha_{k} \quad \text { for each processor } P_{k} \text { with } k \neq i, j
\end{aligned}
$$




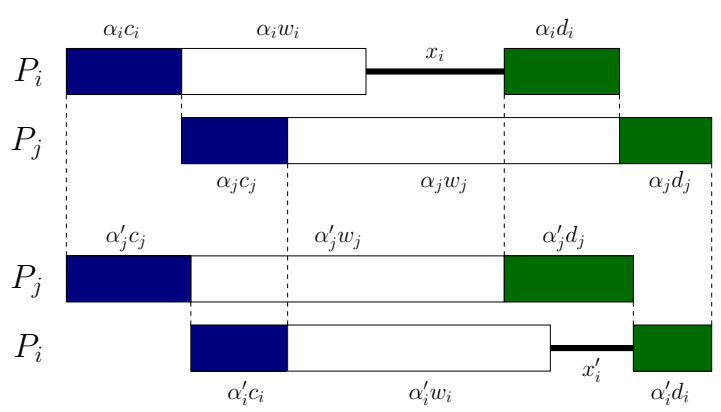

Figure 6. From $\mathcal{S}$ to $\mathcal{S}^{\prime}$, in the case $c_{i}>c_{j}$

Moreover, let us set $x_{k}^{\prime}=0$ for all $k \neq i$ and $x_{i}^{\prime}=$ $x_{i}\left(\frac{w_{j}+d_{j}}{c_{j}+w_{j}}\right)$. As previously, we need to check that the description of the new schedule in Figure 6 is valid:

- communications for $P_{i}, P_{j}$ take the same time in $\mathcal{S}$ and $\mathcal{S}^{\prime}$ :

The time needed for data transfers for $\left(P_{i}, P_{j}\right)$ in $\mathcal{S}^{\prime}$ is

$$
\begin{aligned}
& c_{j} \alpha_{j}^{\prime}+c_{i} \alpha_{i}^{\prime} \\
& =c_{j} \alpha_{j}+c_{j} \frac{\alpha_{i} c_{i}(1-z)}{c_{j}+w_{j}}+c_{i} \alpha_{i}-c_{i} \frac{\alpha_{i} c_{j}(1-z)}{c_{j}+w_{j}} \\
& =c_{j} \alpha_{j}+c_{i} \alpha_{i}
\end{aligned}
$$

which is the time for data transfers for $\left(P_{i}, P_{j}\right)$ in $\mathcal{S}$. Since $d_{i} / c_{i}=d_{j} / c_{j}=z$, the same analysis holds true for output messages.

- $P_{j}$ starts sending output messages in $\mathcal{S}^{\prime}$ at the same date as $P_{i}$ does in $\mathcal{S}$ :

If we assume that the first processor among $P_{i}, P_{j}$ starts receiving data at time 0 , then $P_{j}$ starts sending its results at time

$$
\begin{aligned}
T_{j} & =\alpha_{j}^{\prime} c_{j}+\alpha_{j}^{\prime} w_{j} \\
& =\left(\alpha_{j}+\frac{\alpha_{i} c_{i}(1-z)}{c_{j}+w_{j}}\right) \cdot\left(c_{j}+w_{j}\right) \\
& =\alpha_{j}\left(c_{j}+w_{j}\right)+\alpha_{i}\left(c_{i}-d_{i}\right)
\end{aligned}
$$

But in $\mathcal{S}$, we have $\alpha_{j}\left(c_{j}+w_{j}\right)=\alpha_{i}\left(w_{i}+d_{i}\right)+$ $x_{i}$, so that $T_{j}=\alpha_{i}\left(c_{i}+w_{i}\right)+x_{i}$, which is exactly the date when the output transfer of $P_{i}$ starts in $\mathcal{S}$.

- $P_{i}$ has enough time to process its tasks:

The time between the beginning of the data transfer for $P_{i}$ and the beginning of its out- put transfer in $\mathcal{S}^{\prime}$ is given by

$$
\begin{aligned}
T_{i}^{2} & =\alpha_{i}^{\prime} c_{i}+\alpha_{i}^{\prime} w_{i}+x_{i}^{\prime} \\
& =\underbrace{\left(\alpha_{i}-\frac{\alpha_{i} c_{j}(1-z)}{c_{j}+w_{j}}\right)}_{\alpha_{i} \frac{w_{j}+d_{j}}{c_{j}+w_{j}}}\left(c_{i}+w_{i}\right)+x_{i}\left(\frac{w_{j}+d_{j}}{c_{j}+w_{j}}\right) \\
& =\frac{w_{j}+d_{j}}{c_{j}+w_{j}}\left(\left(c_{i}+w_{i}\right) \alpha_{i}+x_{i}\right)
\end{aligned}
$$

and the time between the start of $P_{j}$ 's computation and the end of its output transfer in $\mathcal{S}^{\prime}$ is

$$
\begin{aligned}
T_{j}^{2} & =\alpha_{j}^{\prime} w_{j}+\alpha_{j}^{\prime} d_{j} \\
& =\left(\alpha_{j}+\frac{\alpha_{i}\left(c_{i}-d_{i}\right)}{c_{j}+w_{j}}\right)\left(w_{j}+d_{j}\right) .
\end{aligned}
$$

As previously, we have in $\mathcal{S} \alpha_{j}\left(c_{j}+w_{j}\right)=$ $\alpha_{i}\left(w_{i}+d_{i}\right)+x_{i}$, so that we can replace $\alpha_{j}$ by its actual value in the previous equation:

$$
\begin{aligned}
T_{j}^{2} & =\left(\frac{\alpha_{i}\left(w_{i}+d_{i}\right)+x_{i}}{c_{j}+w_{j}}+\frac{\left.\alpha_{i}\left(c_{i}-d_{i}\right)\right)}{c_{j}+w_{j}}\right)\left(w_{j}+d_{j}\right) \\
& =\frac{w_{j}+d_{j}}{c_{j}+w_{j}}\left(\left(c_{i}+w_{i}\right) \alpha_{i}+x_{i}\right)=T_{i}^{2} .
\end{aligned}
$$

Therefore, in $\mathcal{S}^{\prime}$, the end of $P_{j}$ 's output transfer corresponds to the start of $P_{i}$ 's output transfer.

The number of tasks processed in $\mathcal{S}^{\prime}$ is:

$$
\sum_{i} \alpha_{i}^{\prime}=\sum_{i} \alpha_{i}+\frac{\alpha_{i}\left(c_{i}-c_{j}\right)(1-z)}{c_{j}+w_{j}}
$$

Since $c_{i}>c_{j}$, we have built a FIFO one-port schedule that processes more tasks than $\mathcal{S}$, which is in contradiction with the optimality of $\mathcal{S}$, so the case $c_{i}>c_{j}$ never happens.

We apply this approach as many times as needed so that, at the end, the only processor with some idle time is the last enrolled processor $P_{q}$.

We are now able to prove Theorem 1.

Proof. The previous lemmas prove that there exists an optimal FIFO one-port schedule where the only participating processor possibly with idle time is the last one. In order to achieve the proof Theorem 1, we still need to prove that there exists an optimal FIFO schedule where processors are ordered by non-decreasing values of the $c_{i}$ 's.

Let us consider an optimal one-port schedule $\mathcal{S}$ where the last processor only has idle time. We denote 
by $P_{1}, \ldots, P_{q}$ the processors taking part to the computation, in this order. Suppose that processors are not ordered by non-decreasing value of $c_{i}$. Then, there exists an index $k$ such that $c_{k}>c_{k+1}$. We apply the transformation of the second case of the proof of Lemma 2 to processors $P_{i}=P_{k}$ and $P_{j}=P_{k+1}$. Note that this transformation is valid even if there is no idle time for processor $P_{i}\left(x_{i}=0\right)$. In this case we get $x_{i}^{\prime}=0$ since $x_{i}^{\prime}=x_{i}\left(\frac{w_{j}+d_{j}}{c_{j}+w_{j}}\right)$, so that there is no idle time for $P_{i}$ and $P_{j}$ in the new schedule $\mathcal{S}^{\prime}$. However, the total amount of tasks processed in $\mathcal{S}^{\prime}$ is given by

$$
\sum_{i} \alpha_{i}^{\prime}=\sum_{i} \alpha_{i}+\frac{\alpha_{i}\left(c_{i}-c_{j}\right)(1-z)}{c_{j}+w_{j}} .
$$

Since $z<1$ and $c_{i}>c_{j}, \mathcal{S}^{\prime}$ processes strictly more tasks than $\mathcal{S}$, which contradicts the optimality of $\mathcal{S}$. Therefore, in $\mathcal{S}$, processors are ordered by non-decreasing values of the $c_{i}$ 's.

There remains to prove that:

Lemma 3. An optimal FIFO schedule (with resource selection) can be determined in polynomial time.

Proof. Owing to Theorem 1, we know that there exists an optimal FIFO schedule where processors are ordered by non-decreasing values of $c_{i}$, but we do not know the optimal number of enrolled processors. The following algorithm computes in polynomial time the best throughput, and exhibits an optimal schedule:

1. Sort processors by non-decreasing values of the $c_{i}$ 's: $P_{1}, \ldots, P_{p}$.

2. Build a linear program enrolling all $p$ processors, but with an idle-time variable $x_{i}$ for each of them. This requires the resolution (in rational numbers) of a linear program with $2 p$ variables and $3 p+1$ constraints.

3. The solution of the linear program provides the set of participating processors (those such that $\left.\alpha_{i} \neq 0\right)$ and their load.

\subsection{Optimal FIFO throughput on a bus network}

We give an explicit formula for the optimal throughput of a FIFO schedule, when the platform reduces to a bus network:

Theorem 2. The optimal FIFO one-port solution when $c_{i}=c$ and $d_{i}=d$ achieves the throughput

$$
\rho^{o p t}=\min \left\{\frac{1}{c+d}, \frac{\sum_{i=1}^{p} u_{i}}{1+d \sum_{i=1}^{p} u_{i}}\right\}
$$

where $u_{i}=\frac{1}{d+w_{i}} \prod_{j=1}^{i}\left(\frac{d+w_{j}}{c+w_{j}}\right)$. Note that all processors are enrolled in the optimal solution.

Proof. First of all, we prove that for a given FIFO one-port schedule, the throughput $\rho$ is less than

$$
\min \left\{\frac{1}{c+d}, \frac{\sum_{i=1}^{k} u_{i}}{1+\sum_{i=1}^{k} u_{i} d}\right\} .
$$

Let $S$ be a given one-port schedule enrolling workers $P_{1}$ to $P_{q}$, and let $\alpha_{i}$ be the number of load units processed by $P_{i}$. As we target a bus network, sending one load unit from the master to any processor takes a time $c$, whereas receiving one unit from any worker takes a time $d$. So the time needed to send all data from the master is

$$
T_{\text {send }}=\sum_{i=1}^{q} \alpha_{i} c
$$

and the time needed to receive all results is: $T_{\text {recv }}=$ $\sum_{i=1}^{q} \alpha_{i} d$. As the schedule obeys the one-port model, we know that no reception of the master can start until all sends are completed. Thus we get the constraint $T_{\text {send }}+T_{\text {recv }} \leq 1$. Hence:

$$
T_{\text {send }}+T_{\text {recv }}=\sum_{i=1}^{q} \alpha_{i} c+\sum_{i=1}^{q} \alpha_{i} d=\rho(c+d) \leq 1
$$

It remains to prove that $\rho \leq \tilde{\rho}$, where $\tilde{\rho}=\frac{\sum_{i=1}^{k} u_{i}}{1+\sum_{i=1}^{k} u_{i} d}$. Consider a given FIFO one-port schedule $S$ with throughput $\rho$. $S$ can be viewed as a two-port schedule, so its throughput cannot exceed that of the optimal throughput in the two-port model. As shown in $[6,7]$, the optimal two-port solution for a bus network involves all processors and achieves the throughput $\tilde{\rho}$, hence the result.

Then, we prove that their exists a schedule reaching the optimal throughput $\rho^{\text {opt }}$.

We start with an optimal FIFO two-port schedule $S$ whose throughput is $\tilde{\rho}$ and we transform it into a one-port schedule. We consider two cases:

- $\tilde{\rho} \leq \frac{1}{c+d}$. Then there is no overlap between forward and backward communications. The two-port schedule actually is a one-port schedule, whose throughput is $\tilde{\rho}=\rho^{\text {opt }}$.

- $\tilde{\rho} \geq \frac{1}{c+d}$. This is the case with overlap between communication from and to the master, represented in Figure 7. Therefore we have to delay the receptions of return messages until the master has finished sending data (see second schedule on Figure 7$)$. To do so, we add a gap of length $x=\tilde{\rho} \times(c+d)-1$ between the end of the computation and the transmission of the return message on 


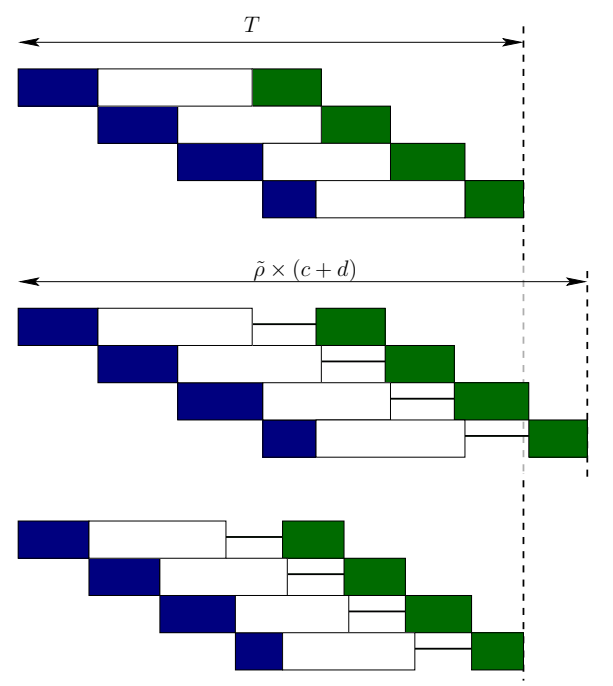

Figure 7. How to transform an optimal two-port schedule into a one-port schedule.

each processor. The overall execution time of the schedule becomes $T^{\prime}=\tilde{\rho} \times(c+d)$. Finally, to obtain a schedule of total execution time 1 , we scale down all quantities by a factor $\frac{1}{\tilde{\rho} \times(c+d)}$ (see the third schedule on Figure 7). We obtain the following characteristics:

number of tasks processed by $P_{i}$ :

$$
\alpha_{i}^{\prime}=\frac{\alpha_{i}}{\tilde{\rho} \times(c+d)}
$$

gap for every processor:

$$
x=1-\frac{1}{\tilde{\rho} \times(c+d)}
$$

The throughput of this new schedule is:

$$
\rho^{\prime}=\frac{\tilde{\rho}}{\tilde{\rho} \times(c+d)}=\frac{1}{c+d}
$$

It remains to prove that the new schedule satisfies the conditions for the one-port model. We will prove that $T_{\text {send }}^{\prime}+T_{\text {recv }}^{\prime} \leq 1$ :

$$
T_{\text {send }}^{\prime}+T_{\text {recv }}^{\prime}=(c+d) \rho^{\prime}=1
$$

Hence this schedule obeys the one-port model, and achieves the bound $\rho^{\text {opt }}$.

\section{MPI experiments}

In this section we present practical tests using oneport LIFO and FIFO strategies. We choose matrix multiplication as the target application to be implemented on a master/worker platform. The multiplication of two matrices results in a single matrix, hence the initial data message will be twice bigger than the output message: $z=1 / 2$. The objective is to minimize the total execution time for executing a large number $M$ of matrix products. In our tests we compare the behavior of the following algorithms:

- a FIFO heuristic using all processors, sorted by non-decreasing values of $c_{i}$ (faster communicating workers first), called INC_C

- a FIFO heuristic using all processors, sorted by non-decreasing values of $w_{i}$ (faster computing workers first), called INC_W

- the optimal one-port LIFO solution, called LIFO.

By construction, the optimal two-port LIFO solution of $[6,7]$ is indeed a one-port schedule. It involves all processors sorted by non-decreasing values of $c_{i}$. These three heuristics are implemented using the linear programming framework developed in Section 2.3: the choice of the heuristic provides both transfer permutations $\sigma_{1}$ and $\sigma_{2}$; the optimal value of the $\alpha_{i}$ 's, as computed by the linear program, are used for the scheduling. The solution of the linear program is expressed in rational numbers, but we need to send, process and return integer numbers of matrices. The policy to round the $\alpha_{i}$ 's to integer values is the following. We first round down every value to the immediate lower integer, and then we distribute the $K$ remaining tasks to the first $K$ workers of the schedule in the order of the sending permutation $\sigma_{1}$, by giving one more matrix to process to each of these workers. For instance with 4 processors $P_{1}$ to $P_{4}$ used in this order for $\sigma_{1}$, if $M=1000, \alpha_{1}=200.4, \alpha_{2}=300.2$, $\alpha_{3}=139.8$ and $\alpha_{4}=359.6$, then $K=2$, and we assign $200+1$ matrices to $P_{1}, 300+1$ to $P_{2}, 139$ to $P_{3}$ and 359 to $P_{4}$. Obviously, rounding induces some load imbalance, which may slightly impact the actual performance of the heuristics.

\subsection{Experimental setting}

All tests were made on the cluster $g d s d m i$, which is located within the LIP laboratory at ENS Lyon, and consists in $\mathrm{P} 42.4 \mathrm{GHz}$ processors with either $256 \mathrm{MB}$ or $1 \mathrm{~GB}$ of memory. In this cluster, 12 nodes are available, so we mainly conduct experiments with one master and 11 workers. To run our test application, we use the MPICH implementation [18] of the Message Passing Interface $M P I$. To solve all linear programs, we used the $l p \_s o l v e$ solver [8]. The total number $M$ of matrices to be processed has been fixed to $M=1000$. 


\subsection{Heterogeneity and linear model}

The cluster described in the previous Section allows us to start with homogeneous conditions: all nodes have the same computation and communicating capabilities. As we target heterogeneous platforms, we simulate heterogeneity by slowing down or speeding up some operation (transfer or computation). We chose to consider the original speed as the slowest available, and sometimes speed up communication or computation when we want to simulate faster workers. For example, to simulate a worker which communicates twice faster than the original speed, we reduce the size of the data and result messages by a factor 2 . The data missing for the computation might appear critical, but we are interested in the execution time rather than in the result, so we randomly fill up all the matrices we use. We proceed in the same manner for the computation: simulating a processor which is 5 times faster is done by processing only a fifth of the original computation amount. We have chosen not to slow down transfers or computations in order to avoid problems of memory swapping, which could have happened with very big message sizes, and also in order to reduce execution time.

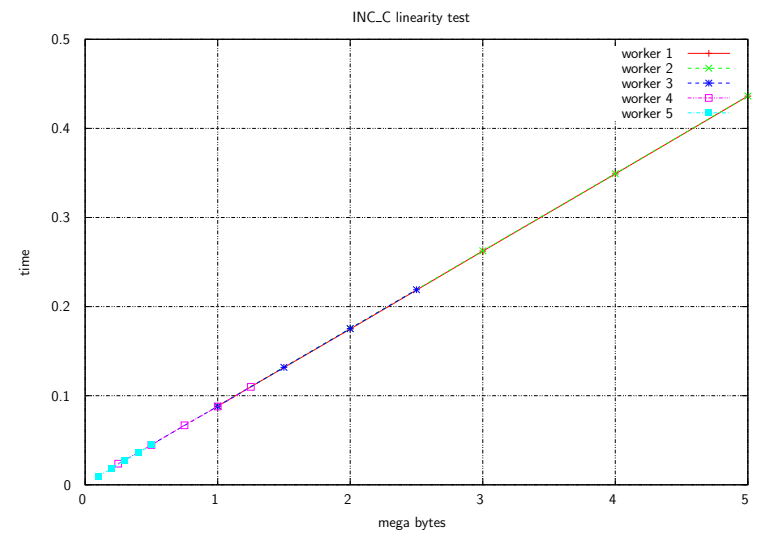

Figure 8. Linearity test with different message sizes, simulating heterogeneous workers.

First, we check that the basic linear cost model which we adopted in our divisible load approach is valid. So we perform a linearity test, by sending different sizes of messages to worker with different (simulated) communication speeds. The results of this test are presented in Figure 8 and show that our assumption on linearity holds true, and that no latency needs to be taken into account.

\subsection{Execution analysis}

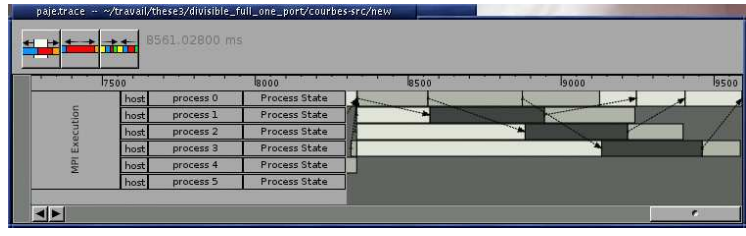

Figure 9. Visualizing an execution on a heterogeneous platform.

To analyze the behavior of the platforms during our experiments, we perform some trace analysis. Figure 9 presents the visualization of one execution, obtained using the Pajé software [13]. The first line represents the activity of the master, while the other five lines represent workers with different speeds. The initialization of the workers is not shown on this figure. The visualization shows the data transfers (in white), the computation (in dark gray) and the output transfers (in pale gray). Note that for each transfer, the bar "starts" when the receiver is ready for communicating, and "ends" when it has received all data. This explains why at the beginning, all workers start receiving, as they are all waiting for the master to send them some data. As the workers of the platform have (simulated) heterogeneous communicating and computing speeds, not necessary all workers are involved in the computation. Indeed, in the execution shown in Figure 9, only the first three workers are actually performing some computation. In this experiment, we use FIFO ordering: the sending order is the same for input data and results.

\subsection{Heuristics comparison}

We present here the results of the experiments for a large number of platforms, randomly generated, with parameters varying from 1 to 10 , where 1 represents the original speed either for communication or for computation, and 10 represents a worker 10 times faster. Each point of the following graphs is obtained as the average performance on 50 platforms randomly generated.

3.4.1. Homogeneous bus platforms. Figure 10(a) shows the experimental results for homogeneous bus platforms: in these tests, all workers have the same communication and computation capabilities.

In this figure, as in the following ones, we plot the actual execution time of each heuristic, after having normalized it against the theoretical prediction for the INC_C heuristic. For example the line "LIFO 
real/INC_C lp" means (execution time of the LIFO heuristic in the experiments)/(theoretical execution time of the INC_C heuristic).

We plot only the INC_C FIFO heuristic because all FIFO strategies are the same with homogeneous communication and computation speeds. In these homogeneous settings, LIFO performs better than FIFO, both in the linear program and in the real experiments.

3.4.2. Heterogeneous bus platforms. Our results on platforms with homogeneous communications and heterogeneous computing speeds are presented on Figure 10(b). This kind of platform corresponds exactly to the platforms used in Theorem 2. The results supports the theoretical study: INC_C gives better results that INC_W. Again, LIFO performs better than the FIFO strategies. Although the experimental results differ from the theoretical prediction, the theory correctly ranks the different heuristics: in the linear programming approach, LIFO is better than INC_C, which is better than INC_W, and this order in the same in the practical experiments.

3.4.3. Heterogeneous star platforms. Figure 10(c) presents the results for the case of platforms with heterogeneous communication speeds and heterogeneous computation capabilities. Again, INC_C is the best FIFO strategy, as predicted by Theorem 1. The results are very similar as for heterogeneous platforms: LIFO is better than the FIFO strategies, and the linear program correctly predicts the relative performance of the heuristics, although absolute performance differ from what is predicted by a factor bounded by $20 \%$.

3.4.4. Changing the communication to computation ratio. In this section, we describe the experiments which we performed to better understand the impact of the ratio between communication and computation cost. Starting from the last set of experiments (Figure 10(c)), we first increase the computation power of each processors by a factor 10, and perform the same experiments. Results are presented in Figure 11(a). The test shows that with small matrix sizes, the performances of the LIFO heuristic are much worse than expected, while the performances of both FIFO strategies are very close to each other. This is quite unexpected as the linear program gives a good performance to the LIFO strategy, as in the previous scenarios. The LIFO heuristic might be very sensitive to small performance variations in this case.

Then, we perform the same experiments with platforms where the communication is 10 times faster than in the original tests, and computation speeds are not changed. Results are presented in Figure 11(b).

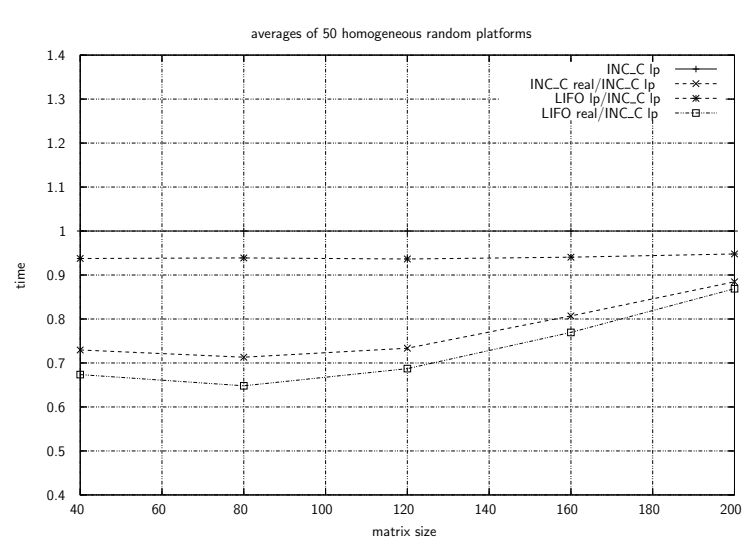

(a) Homogeneous bus performance, normalized by FIFO theoretical running time.

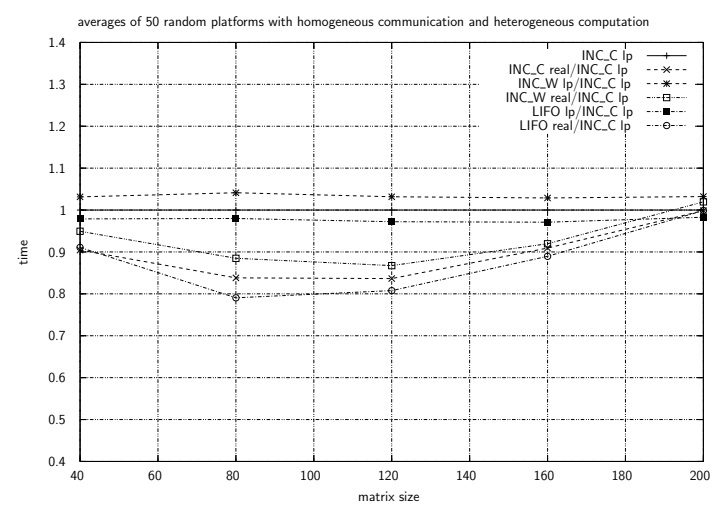

(b) Heterogeneous bus performance, normalized by FIFO theoretical running time.

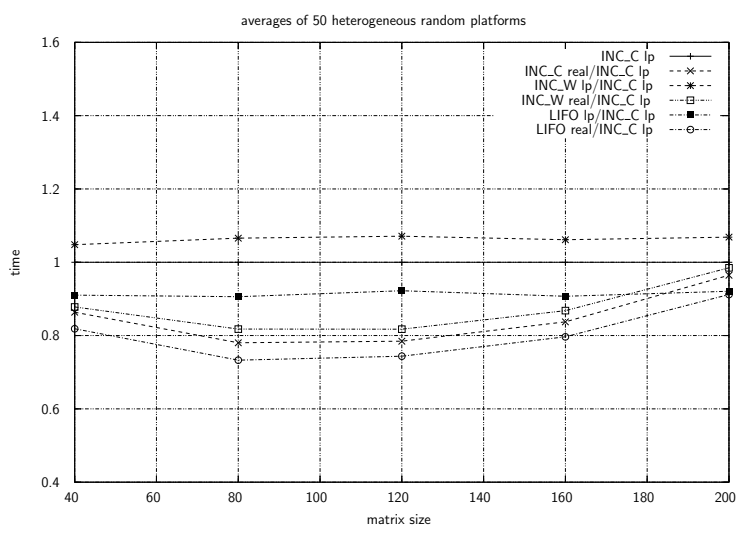

(c) Heterogeneous star performance, normalized by FIFO theoretical running time.

Figure 10. Performance of the heuristics with different experimental settings 


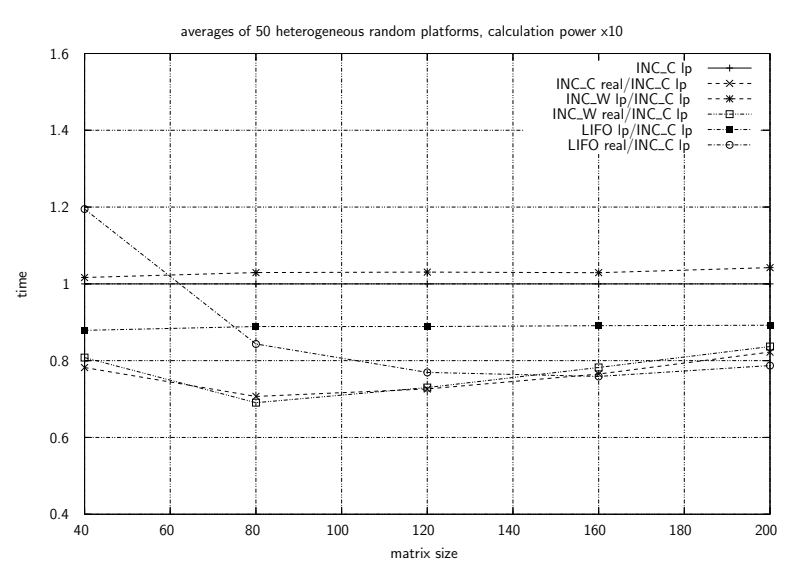

(a) Computation 10 times faster

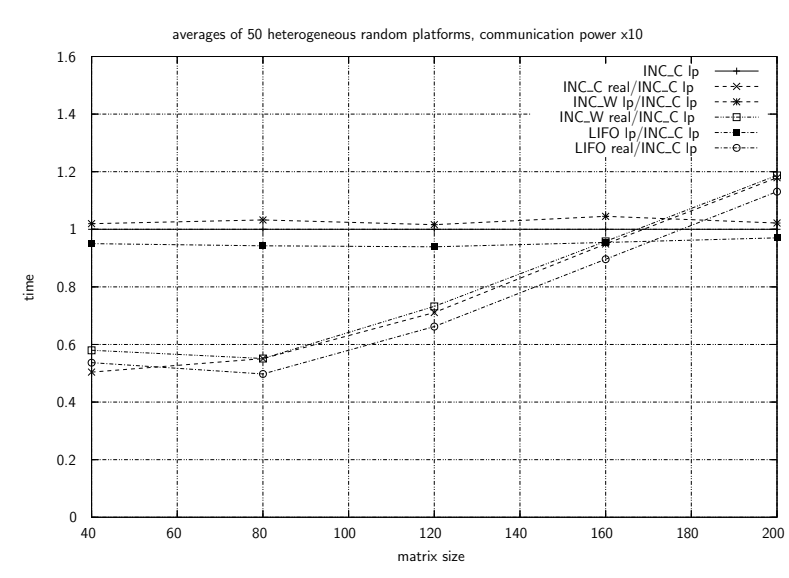

(b) Communication 10 times faster

\section{Figure 11. Heuristics performance for platforms with different communication/computation ratio, nor- malized by FIFO theoretical running time.}

This shows the limits of the linear cost model, as the ratio between practical performance and theoretical throughput increases linearly with the size of the matrices. However the linear program correctly predicts the relative performance of the different heuristics.

\subsubsection{Observing the number of participating} workers. As stated earlier, when considering return messages, it can happen that not all workers should be enrolled in the solution to obtain best performances. In this section, we want to check if our framework correctly determines the optimal number of workers that are involved in the computation. We use a platform consisting in 4 workers, where the first 3 workers are fast both in computation and in communication, and the last worker is slower. The following table precisely describes their characteristics:

\begin{tabular}{|r|c|c|c|c|}
\hline worker: & 1 & 2 & 3 & 4 \\
\hline communication speed: & 10 & 8 & 8 & $x$ \\
\hline computation speed: & 9 & 9 & 10 & 1 \\
\hline
\end{tabular}

Depending on the value of the communication speed $x$ of the last worker, this one should participate or not in the computation. In the following tests, we run our program with a number of slaves from one to four. Then, we record the number of slaves that were really used, and the performance obtained. Figure 12(a) presents the results for $x=1$. In this case, the last worker is never used (even when we authorize four workers to be used). In Figure 12(b), we present the results for the case $x=3$. In this case, the fourth worker is used, and the performance is slightly better when us- ing all four workers (even it is hardly noticeable on the graph). This shows that our framework, on this little example, is able to make the right choice about the number of participating processors.

\section{Related work}

In addition to the landmark book [9], several sources to DLS literature are available: see the two introductory surveys $[10,23]$, the special issue of the Cluster Computing journal entirely devoted to divisible load scheduling [17], and the Web page collecting DLSrelated papers is maintained [22].

DLS applications include linear algebra [12], image processing $[19,21]$, video and multimedia broadcasting $[2,3]$, database searching $[14,11]$, and the processing of large distributed files [26]. These applications are amenable to the simple master-worker programming model and can thus be easily implemented and deployed on computing platforms ranging from small commodity clusters to computational grids [16].

The DLS model comes in two flavors, with a linear cost model and with an affine cost model. The linear cost model is the original model, and has been widely adopted because of its simplicity: several closed-form formulas are available for star, tree, mesh networks among others $[9,22]$. The affine cost model (which amounts to introduce a start-up overhead in the communication cost, and/or in the computation cost) has been advocated more recently, for two main reasons: (i) it is more realistic than the linear model; and (ii) it can- 


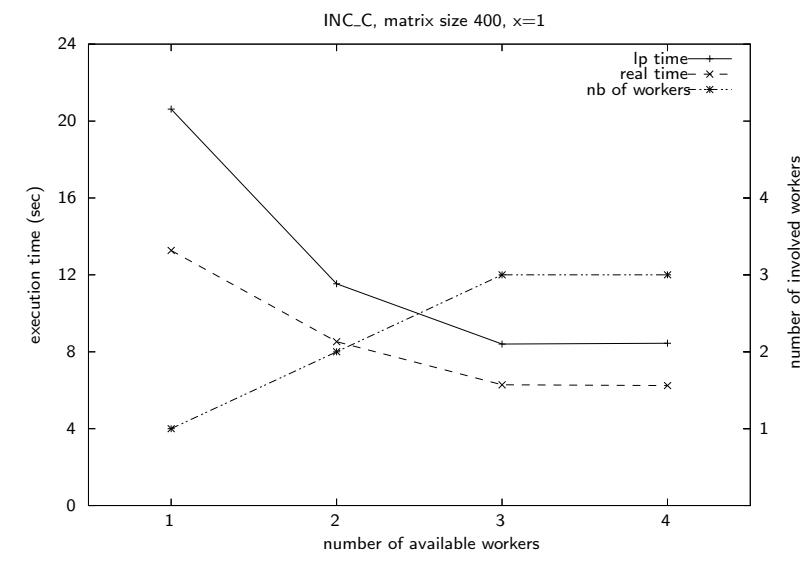

(a) $x=1$

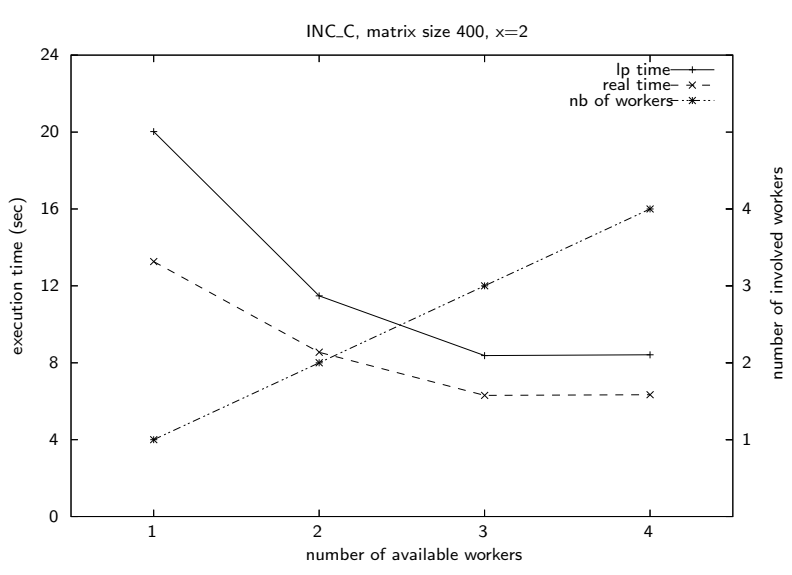

(b) $x=3$

Figure 12. Test of participating workers on a heterogeneous platform.

not be avoided when dealing with multiple-round scenarios, where the master is allowed to send data to the workers with several messages rather than with a single one. Multiple-round strategies are better amenable to pipelining than one-round approaches, but using a linear cost model would then favor sending a large collection of infinitely small messages, hence the need to add communication latencies. However, latencies render the problem more complex: the DLS problem has recently been proved NP-hard on a star network with the affine model [20].

When dealing with one-round scenarios, as in this paper, the linear model is more realistic, especially when the total work to be distributed to the slaves is large. From a theoretical perspective, one major question was to determine whether adding return messages, while retaining the linear model, would keep the DLS scheduling problem polynomially tractable. We failed to answer this question, but we have been able to characterize optimal solutions for FIFO strategies under the one-port model. This result nicely complements our previous study under the two-port model.

Relatively few papers have considered adding return messages in the study of DLS problems. Pioneering results are reported by Barlas [4], who tackles the same problem as in this paper (one round, star platform) but with an affine framework model. Barlas [4] concentrates on two particular cases: one called query processing, where communication time (both for initial and return messages) is a constant independent of the message size, and the other called image processing, which reduces to linear communication times on a bus network, but with affine computation times. In both cases, the optimal sequence of messages is given, and a closed-form solution to the DLS problem is derived. In [15], the authors consider experimental validation of the DLS model for several applications (pattern searching, graph coloring, compression and join operations in databases). They consider both FIFO and LIFO distributions, but they do not discuss communication ordering.

Rosenberg [24] and Adler, Gong and Rosenberg [1] also tackle the DLS model with return messages, but they limit themselves to a bus network (same link bandwidth for all workers). They introduce a very detailed communication model, but they state results for affine communication costs and linear computation costs. They have the additional hypothesis that worker processors can be slowed down instead of working at full speed, which allows them to consider no idle times between the end of the execution and the emission of the return messages. They state the very interesting result that all FIFO strategies are equivalent, and that they perform better than any other protocol. Note that our results, although not derived under the same model, are in accordance with these results: when the star platform reduces to a bus platform, the theoretical results of this paper show that all processors should be involved in the computation, and that their ordering has no impact on the quality of the solution.

Finally, we point out that Altilar and Paker [3] also investigate the DLS problem on a star network, but their paper is devoted to the asymptotic study of several multi-round strategies. 


\section{Conclusion}

In this paper, we present two optimality results for scheduling divisible load on star networks in presence of return messages and under the one-port model. First we are able to characterize the optimal FIFO scheduling on general star networks, where fast-communicating processors should be enrolled first in the computation. We also provide an analytic expression of the total amount of load that can be processed by a FIFO scheduling on homogeneous networks. We have also provided a set of MPI experiments to assess the performance of several heuristics in a real framework.

This paper constitutes the first attempt, to the best of our knowledge, to take return messages into account under the one-port model. Nevertheless, we are still far from the optimal solution of the general problem. Indeed, we are only able to provide optimal schedulings for fixed communication orderings such as FIFO or LIFO. The complexity of finding the pair of optimal permutations for forward and return messages remains open, under both the one-port and two-port models. Despite the simplicity of the linear cost model both for computations and communications, the problem looks very combinatorial, and we conjecture that it is $\mathrm{NP}$ hard.

\section{References}

[1] M. Adler, Y. Gong, and A. L. Rosenberg. Optimal sharing of bags of tasks in heterogeneous clusters. In 15th ACM Symp. on Parallelism in Algorithms and Architectures (SPAA'03), pages 1-10. ACM Press, 2003.

[2] D. Altilar and Y. Paker. An optimal scheduling algorithm for parallel video processing. In IEEE Int. Conference on Multimedia Computing and Systems. IEEE Computer Society Press, 1998.

[3] D. Altilar and Y. Paker. Optimal scheduling algorithms for communication constrained parallel processing. In Euro-Par 2002, LNCS 2400, pages 197-206. Springer Verlag, 2002.

[4] G. Barlas. Collection-aware optimum sequencing of operations and closed-form solutions for the distribution of a divisible load on arbitrary processor trees. IEEE Trans. Parallel Distributed Systems, 9(5):429 441, 1998.

[5] O. Beaumont, L. Marchal, V. Rehn, and Y. Robert. Fifo scheduling of divisible loads with return messages under the one-port model. Research Report 200552, LIP, ENS Lyon, France, Oct. 2005. Available at graal.ens-lyon.fr/ yrobert/.

[6] O. Beaumont, L. Marchal, and Y. Robert. Scheduling divisible loads with return messages on heterogeneous master-worker platforms. In International Conference on High Performance Computing (HiPC), LNCS, Goa, India, 2005. Springer.

[7] O. Beaumont, L. Marchal, and Y. Robert. Scheduling divisible loads with return messages on heterogeneous master-worker platforms. Research Report 2005-21, LIP, ENS Lyon, France, may 2005. Available at www.ens-lyon.fr/LIP/Pub/Rapports/RR/RR2005/ RR2005-21.pdf.

[8] M. Berkelaar. LP_SOLVE. http://www.cs. sunysb.edu/ algorith/implement/lpsolve/ implement.shtml.

[9] V. Bharadwaj, D. Ghose, V. Mani, and T. Robertazzi. Scheduling Divisible Loads in Parallel and Distributed Systems. IEEE Computer Society Press, 1996.

[10] V. Bharadwaj, D. Ghose, and T. Robertazzi. Divisible load theory: a new paradigm for load scheduling in distributed systems. Cluster Computing, 6(1):7-17, 2003.

[11] J. Blazewicz, M. Drozdowski, and M. Markiewicz. Divisible task scheduling - concept and verification. Parallel Computing, 25:87-98, 1999.

[12] S. Chan, V. Bharadwaj, and D. Ghose. Large matrixvector products on distributed bus networks with communication delays using the divisible load paradigm: performance and simulation. Mathematics and Computers in Simulation, 58:71-92, 2001.

[13] J. C. de Kergommeaux, B. de Oliveira Stein, and P.-E. Bernard. Pajé, an interactive visualization tool for tuning multi-threaded parallel applications. Parallel Computing, 26(10):1253-1274, 2000.

[14] M. Drozdowski. Selected problems of scheduling tasks in multiprocessor computing systems. PhD thesis, Instytut Informatyki Politechnika Poznanska, Poznan, 1997.

[15] M. Drozdowski and P. Wolniewicz. Experiments with scheduling divisible tasks in clusters of workstations. In Proceedings of Euro-Par 2000: Parallel Processing, LNCS 1900, pages 311-319. Springer, 2000.

[16] I. Foster and C. Kesselman, editors. The Grid: Blueprint for a New Computing Infrastructure. Morgan Kaufmann Publishers, Inc., San Francisco, USA, 1999.

[17] D. Ghose and T. Robertazzi, editors. Special issue on Divisible Load Scheduling. Cluster Computing, 6, 1, 2003.

[18] W. Gropp, E. Lusk, N. Doss, and A. Skjellum. A highperformance, portable implementation of the MPI message passing interface standard. Parallel Computing, 22(6):789-828, Sept. 1996. see also http: //www-unix.mcs.anl.gov/mpi/mpich/.

[19] C. Lee and M. Hamdi. Parallel image processing applications on a network of workstations. Parallel Computing, 21:137-160, 1995.

[20] A. Legrand, Y. Yang, and H. Casanova. Npcompleteness of the divisible load scheduling problem on heterogeneous star platforms with affine costs. Research Report CS2005-0818, GRAIL Project, University of California at San Diego, march 2005. 
[21] X. Li, V. Bharadwaj, and C. Ko. Distributed image processing on a network of workstations. Int. J. Computers and Applications (ACTA Press), 25(2):110, 2003.

[22] T. Robertazzi. Divisible Load Scheduling. http: //www.ece.sunysb.edu/ tom/dlt.html.

[23] T. Robertazzi. Ten reasons to use divisible load theory. IEEE Computer, 36(5):63-68, 2003.

[24] A. L. Rosenberg. Sharing partitionable workloads in heterogeneous NOws: greedier is not better. In Cluster Computing 2001, pages 124-131. IEEE Computer Society Press, 2001.

[25] A. Schrijver. Theory of Linear and Integer Programming. John Wiley \& Sons, New York, 1986.

[26] R. Wang, A. Krishnamurthy, R. Martin, T. Anderson, and D. Culler. Modeling communication pipeline latency. In Measurement and Modeling of Computer Systems (SIGMETRICS'98), pages 22-32. ACM Press, 1998.

\section{Biographies}

Olivier Beaumont received the $\mathrm{PhD}$ degree from the Université de Rennes in 1999. He is currently an associate professor in the LaBRI laboratory in Bordeaux. His main research interests are parallel algorithms on distributed memory architectures.

Loris Marchal is currently a $\mathrm{PhD}$ student in the LIP laboratory at ENS Lyon. He is mainly interested in parallel algorithm design for heterogeneous platforms and in scheduling techniques. He is a student member of the IEEE and the IEEE Computer Society.

Veronika Rehn is currently a Master student in the LIP laboratory at ENS Lyon. She is mainly interested in parallel algorithm and divisible load scheduling.

Yves Robert received the $\mathrm{PhD}$ degree from Institut National Polytechnique de Grenoble in 1986. He is currently a full professor in the Computer Science Laboratory LIP at ENS Lyon. He is the author of four books, 95 papers published in international journals, and 120 papers published in international conferences. His main research interests are scheduling techniques and parallel algorithms for clusters and grids. He is a Fellow of the IEEE. 